\title{
CHEN Wei's Experience in the Treatment of Postoperative Cough of Lung Cancer from Qi, Blood and Water
}

\author{
Lulu Liu ${ }^{1}$, Wei Chen ${ }^{2} *$ \\ ${ }^{1}$ Graduate School of Anhui University of Chinese Medicine, \\ Hefei 230000, Anhui, China \\ ${ }^{2}$ Department of Respiratory Medicine, The First Affiliated Hospital of Anhui University of Chinese Medicine, \\ Hefei 230000, Anhui, China \\ * Correspondence Author
}

\begin{abstract}
To summarize Chen Wei's experience in treating postoperative cough of lung cancer. Teacher Chen holding the view that that Lung loses the government and management of regulation and failure and the imbalance of qi, blood and body fluid are the key pathogenesis of cough after lung cancer surgery. Therefore, from the basic prescription of treating qi, blood and water, teacher Chen's thinking of traditional Chinese medicine of inheriting essence and keeping innovation is briefly described. The treatment of qi includes absorbing the innate essence Qi, supplementing the acquired zongqi, and strengthening the guard qi on the body surface. The treatment of blood includes supplementing the blood of the body and evacuating the blood stasis after operation. The treatment of water includes nourishing body fluid of lungs and removing phlegm. Taking a case as an example, it is analyzed that the physiological characteristics that one loss is all loss and one glory is all glory of qi, blood and body fluid are the main reason for the doubling of drug half effect.
\end{abstract}

Keywords: CHEN Wei, postoperative cough of Lung Cancer, Lung loses the government and management of regulation and failure, Qi, Blood and water, Experience.

\section{Introduction}

Lung cancer is China's highest incidence rate and mortality rate of malignant tumor[1]. With the increasing attention of people to their own health and the gradual progress of modern medical technology, the detection rate of early lung cancer has been continuously improved, and surgical resection is one of the main treatment methods. And surgical treatment of non-small cell lung cancer is usually considered to be the most basic treatment in the early stage[2]. However, $25 \%-50 \%$ of patients will cough for a long time after lung cancer surgery, which will significantly reduce the quality of life of patients[3].

Chen Wei is the first young Qihuang scholar of the State Administration of traditional Chinese medicine, the chief physician of the Department of respiratory medicine of the First Affiliated Hospital of Anhui University of traditional Chinese medicine, the successor of the fifth batch of old Chinese medicine academic experience of the country, a teacher of the first national famous Chinese Medicine Professor Han Mingxiang, and the person in charge of the famous Chinese medicine studio in Anhui Province. Chen believes that TCM broad and profound, adhere to the essence of inheritance and innovation, but the road to Jane is nothing more than "cure for the root" four words. "Lung governing Festival" is a high generalization of the physiological relationship between Qi, blood, body fluid and the organs, tissues and organs of the lung and the whole body. Therefore, the etiology and pathogenesis of cough after lung cancer surgery cannot be separated from the three aspects of "Qi, blood and water". The regulation of the lung on the relationship between Qi, blood and body fluid can be regarded as the three corners of the triangle. Only when the three corners are fixed, the triangle can be stable, that is, the physiological function of the lung can be brought into normal play[4]. The author has the honor to accompany the doctor and witness the whole process of traditional Chinese medicine treatment to make the patient "lung retreatment section, triangle stability and cough self-healing". Chen Shi's experience in treating postoperative cough of lung cancer is summarized as follows.

\section{Etiology and Pathogenesis of Cough after Lung Cancer Surgery}

After lung cancer surgery, the accumulation and elimination in the lungs of patients still have long-term cough, which is nothing more than cough due to Qi, cough due to blood and cough due to water. Under the continuous invasion of tumor growth and surgical trauma, the lungs of the body suffer from loss of Qi, blood and body fluid, and fatigue[5]. The normal function of the lung depends on the benign cycle interaction between air, blood and water. However, when the lung is out of treatment, it will turn into the pathological product of the vicious circle of the three. The lung is out of nourishment, the zongqi is insufficient, the xuansu is abnormal, resulting in Qi deficiency and blood stasis; The rise and fall of Qi machine is unbalanced, the distribution of body fluid and blood operation cannot be carried out normally, the body fluid stops into phlegm, the blood stops into blood stasis, and the accumulation of phlegm and blood stasis hinders the Qi machine. Its etiology and pathogenesis can be divided into the following aspects.

\subsection{Deficiency of Vital Qi and Loss of Lung Qi}

"All Qi belong to the lung". Yiyuan points out that the main causes of cough are nothing more than "stagnation of Qi, inversion of Qi and deficiency of Qi". There are more patients 
with lung cancer after operation than after activity, and wheezing is obvious, just as the "lung deficiency, less breath and wheezing" put forward in the criterion of syndrome and treatment. Chen Shi believes that the cause of cough and asthma must be the propaganda and distribution of the lung, the elimination and reduction of dysfunction, and the correction of its essence must be caused by the evil of deficiency and reality. However, most patients with lung cancer are due to the lack of healthy qi, which leads to the accumulation of evil and toxin in the lung, which is always based on deficiency and excess. After lung cancer surgery, the volume of lung decreases, the rise and fall of Lung Qi are disturbed, and the accumulation of excess and evil has been eliminated, which is mostly due to the syndrome of qi deficiency. Therefore, postoperative patients with lung cancer often suffer from low cough sound, shortness of breath, fatigue, spontaneous sweating, cough due to pathogenic factors, as well as impaired spleen and stomach function such as nausea and vomiting and fullness of epigastric and abdominal distension[6].

\subsection{Blood Stasis Does not Change and New Blood Does not Grow}

Lung cancer belongs to the category of "lung accumulation" in traditional Chinese medicine, and the disease lies in blood fraction. "Blood syndrome theory·cough" said: "if there is blood stasis in it, it will hinder the airway and cannot rise or fall. If there is Qi obstruction, there is water obstruction, and water obstruction is phlegm drinking." However, the lung was injured by the golden blade, and the vessels in the lung were damaged and bled, which became the blood of menstruation. The blood of menstruation was not discharged out of the body or dissipated in time, leaving it in the body as blood stasis[7]. The lung loses blood, the heart and spleen lose nourishment, and the blood is unable to flow. It stops and becomes blood stasis. Therefore, the patient may have chest tingling, tongue ecchymosis, sublingual vein bruise, blue and purple, with dull complexion and lip color, etc.

\subsection{Stagnant Water and Body Fluid Consumption Injury}

The body fluid of the human body is transmitted from the spleen to the lung, and is distributed to the whole body and the bladder through the propaganda and elimination of the lung. The lung is the source of water, the lung qi is insufficient and cannot be transported to the four cloth of Tianjin, the spleen deficiency is not transported, the water dampness stops gathering, turns into phlegm and drinks, and is stored in the lung. If the lung qi is unfavorable, there must be cough and asthma. In addition, the mother's disease will affect her son for a long time, resulting in no golden water. The essence of traditional Chinese medicine of tumor is heat toxin, which is most likely to burn the body fluid. The deficiency of kidney yin and stomach fluid can not nourish the lung gold, which is contrary to the physiological characteristics of lung like moistening and evil dryness. Therefore, patients can see expectoration, anorexia, body emaciation, dry fur and other symptoms.

\section{Treatment from "Qi"}

\subsection{Absorb the Innate Essence and Qi}

Ancient doctors believed that the essence and Qi became stars in the sky and grains in the earth. The combination of the essence and Qi of heaven and earth is the origin of all things. Human life and spirit are based on essence and Qi[8]. The kidney mainly stores and seals the essence of Qi, which is the foundation of congenital. It has the function of absorbing the clear Qi inhaled by the lung and maintaining the depth of inhalation. Clinical patients with lung cancer after surgery often have shallow breathing. Chen believes that breathing is propaganda and inhalation is depression, in which the absorption of kidney qi is indispensable. Therefore, we can see the importance of absorbing innate essence and Qi. At this time, the prescription needs to distinguish between yin and Yang. If there are symptoms such as fear of cold, clear and long urination, frequent urination at night, weak waist and knee and light and fat tongue, add and subtract Jinkui Shenqi Pill, dry Rehmannia glutinosa, Cornus officinalis and yam to nourish yin and essence, and take the purpose of "seeking Yang from Yin"; The deficiency of Nourishing Yin can invigorate the kidney yang and restore normal Qi[9]. If it is accompanied by night sweats, spermatorrhea, hot hands and feet, dry tongue, sore throat, red tongue and less moss, it is necessary to add and subtract qiweiduqi pill to cultivate the deficiency of Yin essence of Tonifying the kidney. Schisandra chinensis in the prescription not only astringes the lung and absorbs Qi, but also astringes essence and stops the residue.

\subsection{Tonifying the Acquired Zongqi}

Zongqi is formed by the combination of natural clear Qi and valley Qi. In nature, Qi clearing depends on the inhalation of the lung. Valley Qi is produced by the essence of water and valley transported by the spleen and stomach. Therefore, zongqi belongs to the category of acquired Qi. Chen Shi believes that a long illness will hinder zongqi. The etiology and pathogenesis of postoperative cough of lung cancer are mixed with deficiency and excess, mainly deficiency, which should be attributed to internal injury cough. Spleen deficiency leads to healthy movement, dereliction of duty and failure to transfer body fluid. Lung deficiency leads to abnormal xuanjiang without dispersing body fluid. Water and fluid imbalance, phlegm and dampness stop, and then cough and spit[10]. Therefore, in the treatment of internal injury cough, attention should be paid to protecting the Qi of the spleen and stomach, that is, tonifying the zongqi after the day. Professor Hong Guangxiang, a master of Chinese medicine, clearly pointed out in clinical practice that Buzhong Yiqi Decoction is the core prescription for tonifying zongqi. Astragalus membranaceus in the prescription is the best medicine for tonifying zongqi. If the lung Spleen Qi is strong, the zongqi source is sufficient[11]. This prescription not only makes the acquired biochemistry active, but also can lift the middle Qi and restore the function of the spleen and stomach as the hub of Qi lifting[12].

\subsection{Gas Protection of Solid Body Surface}

"Su Wen" said: "the defender, the fierce Qi of water and valley." Wei Qi can resist external evil, warm the whole body and adjust the reason. The long-term and stable foundation of cough disease after lung cancer surgery is inseparable from solid protective Qi. Chen Shi believes that Yupingfeng powder is the best prescription for strengthening protective Qi, 
especially for patients with corresponding symptoms such as repeated cough, fear of cold and sweating after lung cancer surgery. Modern experimental studies have proved that Yupingfeng powder can exert the effects of immune regulation, anti-inflammatory, bacteriostasis and anti-tumor through a variety of mechanisms[13]. Astragalus membranaceus, Fangfeng and Atractylodes macrocephala can make the body clear and dense, dispel evil without hurting the right, and close the door without leaving enemies.

\subsection{Gas Machine for Connecting and Lowering Triple Energizer}

The cause of cough is not only the deficiency of external Qi but also the reverse of Qi. The movement balance of Qi is first manifested in that the lung resides on the diaphragm and its Qi is subdued; The liver is located under the diaphragm and its Qi rises; The Qi mechanism of the whole body is coordinated by the liver and lung, followed by the spleen and stomach as the hub of the rise and fall of the Qi mechanism of the human body, and the collateral relationship between the lung and the meridians of the large intestine. Therefore, the failure of lung qi is the main factor, and the failure of Three Jiao Qi mechanism such as disharmony between spleen and stomach and stagnation of liver Qi will aggravate cough[14]. For example, Xuanfu Daizhe Decoction can not only reduce retrograde phlegm, but also replenish qi and stomach. It is actually used to treat Qi retrograde cough caused by deficiency and failure of middle Jiao after lung cancer surgery.

\section{Treatment from "Blood"}

\subsection{Nourish the Blood of the Body}

The malignant tumor starts occult and gradually consumes the blood in the patient's body. In addition, the inevitable vascular injury during the operation makes the blood flow out of the body without its way. Therefore, the state of deficiency and stagnation of blood circulation in patients is difficult to recover quickly. It can be seen that it is necessary to supplement blood circulation with traditional Chinese medicine. Chen Shi often uses the addition and subtraction of Siwu Decoction, and occasionally sees patients with blood in sputum. Chen Shi believes that the cause is that Qi does not absorb blood, so he makes good use of the addition and subtraction of Guipi Decoction to control the blood of lung collaterals in the pulse. If the patient is complicated with non infectious low fever, Danggui Buxue Decoction is used, and the clinical effect is quite good.

\subsection{Evacuation of Postoperative Blood Stasis}

Chen Shi believes that pulmonary accumulation must have blood stasis, and accumulation cannot be achieved without blood stasis. For example, the Yellow Emperor's Canon of internal medicine says, "if you can't warm your Qi, you can't disperse your blood coagulation inside, and your body fluid is astringent and seeping. If you don't go away, you can accumulate it." And after the operation, due to the vascular injury in the patient, the body automatically turned on the self-healing mode, mostly in a hypercoagulable state. Therefore, Chen Shi often uses Taohong Siwu Decoction to add and subtract, treat deficiency and blood stasis at the same time, replenish the middle line in the whole prescription, nourish blood but not stagnate blood; The whole prescription plays the effects of promoting blood circulation, promoting Qi, supporting righteousness and eliminating evil[15]. In addition, Guizhi Fuling pill can disperse blood stasis without harming the health. It can be used for a variety of diseases. However, TCM syndrome differentiation and treatment often have "simultaneous treatment of different diseases" and can also be used for postoperative patients with lung cancer[16].

\section{Treatment from "Water"}

\subsection{Body Fluid Nourishing Lung Collaterals}

The metaplasia and resection of pneumocele both hurt the body fluid and consume gas, and the internal consumption of yin and fluid, so it is often dominated by dry cough. However, according to the physiological characteristics of lung liking moistening and hating dryness, traditional Chinese medicine that nourishes the body fluid of lung collaterals is essential in the prescription. Shashen Maidong decoction has the function of clearing and nourishing the lung and stomach, generating fluid and moistening dryness, and relaxing dryness and heat, so that the Yin of the lung and stomach can be supplemented, the fluid of the lung collaterals can be maintained, and the dry cough can be eliminated. The medicine of the whole prescription is light and not greasy, and the disease position of the treated syndrome is partial to the upper coke. Chen Shi commonly used and the Chinese medicine in this prescription. Radix Adenophorae can clear and nourish lung qi, and Radix Ophiopogonis can moisten lung orifices. It is an important medicine for clearing gold and protecting lung[17].

\subsection{Phlegm and Drinking in Buhua Waterway}

Repeated damage to the lung makes the body fluid lose its function. In addition, the child disease and the mother are endogenous with water dampness, which all turn into phlegm and drink. Phlegm drink is divided into tangible and intangible. The former coughs with sputum, and the latter is hidden in the lung, which often makes patients consciously cough with phlegm in the pharynx. "A person's airway is always smooth. If it is smooth, the body fluid will flow, and there will be no trouble of phlegm and drinking." The former master Chen used to use the second of "all phlegm and drink are precious" Chen soup as the basic recipe. For the latter, if qi depression is the main consideration, Banxia Houpu decoction is considered, and if phlegm obstruction is the main consideration, Fritillaria Gualou powder is considered.

\section{Examples of Medical Records}

Zhao, male, 63 years old, first diagnosed on December 14, 2021. On October 11, 2021, the patient underwent radical resection of micro invasive adenocarcinoma of the right upper lung in an external hospital. After the operation, he coughed repeatedly for 2 months, recurred and aggravated for 3 days, and went to our teacher's clinic. Four diagnoses: the patient coughs, coughs a small amount of yellow sticky phlegm, pharyngeal itching, accompanied by fatigue, wheezing after activity, occasional chest tingling, acceptable sleep, short red urine and dry stool; The tongue is red and less fluid, the moss 
is thin and yellow, and the pulse is fine and smooth.

Treatment: clearing lung and resolving phlegm, supplementing qi and relieving asthma, removing blood stasis and dredging collaterals. Prescription: dangshen 10g, Ganoderma lucidum $10 \mathrm{~g}$, aster $10 \mathrm{~g}$, winter flower $10 \mathrm{~g}$, bitter almond 9g, Terminalia chebula $20 \mathrm{~g}$, Tingli $10 \mathrm{~g}$ cloth bag, loquat leaf $20 \mathrm{~g}$, roasted mulberry white skin $30 \mathrm{~g}$, winter melon seed $30 \mathrm{~g}$, Zhuru $10 \mathrm{~g}$, Scutellaria $15 \mathrm{~g}$, earthworm $10 \mathrm{~g}$, reed root $20 \mathrm{~g}$, orange $10 \mathrm{~g}$, Bombyx mori $10 \mathrm{~g}$, corydalis $10 \mathrm{~g}$, roasted licorice $6 \mathrm{~g}$, a total of 7 doses, decocted in water, one dose a day, taken separately in the morning and evening.

At the second visit on December 21, 2021, the patient's cough frequency was reduced, white sticky sputum was coughed, wheezing was improved after activity, and there was still fatigue; the tongue is red, the moss is thin and greasy, and the pulse is fine and smooth. Prescription: $15 \mathrm{~g}$ Scutellaria baicalensis, $10 \mathrm{~g}$ Poria cocos, $10 \mathrm{~g}$ Atractylodes macrocephala and $10 \mathrm{~g}$ Ophiopogon japonicus were added to the first diagnosis formula, and 7 doses were added.

On December 28, 2021, the patient complained that the symptoms of cough, fatigue and asthma were significantly improved, and the urination was slightly short and red; the tongue is slightly red, the moss is thin and white, and the pulse is fine and smooth. Prescription: two diagnostic prescriptions: orange, corydalis, Zhuru; Add Cheqinazi $10 \mathrm{~g}$ cloth bag, $8 \mathrm{~g}$ Shiwei and $8 \mathrm{~g}$ angelica, and add 7 doses. After telephone follow-up, the symptoms of cough and asthma were significantly improved.

According to this medical record, the main symptom of patients is recurrent cough after lung cancer surgery. The formation of pulmonary accumulation does not happen overnight, but is the result of the internal deficiency of positive Qi, phlegm, blood stasis and heat toxin[18]. Chen Shi believes that the patient with chronic illness is not pure deficiency and no evil. Now the patient's cough is aggravated again. The essence is deficiency and excess mixed with syndrome. The prescription should treat both symptoms and symptoms and attack and supplement. The patient's cough, fatigue and wheezing after activity indicate that the patient has the disadvantage of lung qi purging on the basis of deficiency of Qi and blood after operation; Chest tingling indicates pulmonary collateral stasis; There are yellow phlegm, short red urine, dry stool, red tongue and less fluid, which reflect the lack of body fluid of the patient; The above symptoms can be summarized into three aspects: the imbalance of "Qi, blood and water".

Our teacher's first diagnostic prescription, Codonopsis pilosula and Ganoderma lucidum are used to replenish the Qi of lung and spleen; Aster, butterbur, Terminalia chebula and almond can restrain lung, reduce Qi and relieve cough; Yanhusuo can evacuate the blood stasis after operation and alleviate the pain of sharp blade; Reed roots can nourish the body fluid of lung collaterals, loquat leaves, mulberry bark, white gourd seeds, bamboo Ru and Scutellaria baicalensis to clear the hot phlegm of waterways; Assist orange red to smooth the Qi machine of the whole body to help the operation of body fluid and blood; The patient's pharyngeal itching suggested that the wind evil entered the lung, so the stiff silkworm was given to expel the wind evil in vitro. "When the lung qi moves, it will cough, when the spleen is wet, and when the lung and spleen move, it will cough". Our teacher believes that cough should pay attention to the simultaneous treatment of lung and spleen, and patients should be based on deficiency after operation. However, in order to avoid closing the door and leaving bandits in the first diagnosis, we should use Poria cocos and Atractylodes macrocephala to strengthen the spleen and replenish qi in the second diagnosis. Considering the bitter cold of Scutellaria baicalensis, it is easy to damage the spleen and stomach after taking it for a long time; Tracing back to the source, the patient is due to the deficiency of lung yin, the inflammation of deficiency fire, burning fluid as phlegm, which is manifested as yin deficiency and phlegm fire, so Ophiopogon japonicus is added to nourish yin and moisten the lung. During the three diagnosis, the residual heat of patients is not cleared, and the urination of Cheqianzi and Shiwei is added to dissipate the residual heat. Angelica can nourish blood, promote blood circulation and strengthen its foundation, so that the whole party can abide by the principle of both attack and supplement and the harmony between deficiency and reality. These drugs perform their duties on the surface. However, due to the physiological characteristics of "one loss and one prosperity" of Qi, blood and body fluid, this prescription has achieved the therapeutic effect of half the effect of the drug.

\section{Acknowledgement}

This paper is supported by the projects: talent support project for young Qihuang scholars of the State Administration of traditional Chinese medicine (Chinese medicine renjiaofa[2020] No.7); Supported by the youth fund of the National Natural Science Foundation of China (fund No.81704060); General fund support project of Anhui Natural Science Foundation Committee (fund No.1808085MH264).

\section{References}

[1] He Jie, Li Ni, Chen Wanqing, Wu Ning, Shen Hongbing, Jiang Yu, Li Jiang, Wang Fei, Tian Jinhui. Guidelines for lung cancer screening and early diagnosis and treatment in China (2021, Beijing)[J]. Chinese Journal of Oncology, 2021, 30 (02): 81-111.

[2] Khan S, Ali S, Muhammad. Exhaustive Review on Lung Cancers: Novel Technologies[J]. Curr Med Imaging Rev, 2019, 15(9): 873-883.

[3] Xie MR, Zhu YF, Zhou MQ, et al. Analysis of factors related to chronic cough after lung cancer surgery[J]. Thorac Cancer, 2019, 10(4): 898-903.

[4] Zheng Lili Li, Li Zegeng. To explore the etiology and pathogenesis of chronic obstructive pulmonary disease from the "lung governing Festival" $[\mathrm{J}]$. Journal of Jiangxi University of Traditional Chinese Medicine, 2017, 29 (04): $1-3+8$.

[5] Wang Fang, Hou Qiuyu, Wang Shiai, Li Mingxin. Study on TCM syndrome characteristics and treatment of chronic cough after lung cancer surgery[J]. Clinical Research of Traditional Chinese Medicine, 2018, 10(12): 98-100.

[6] Xu Yanchao, Li Honglin, Li Jilei, Ma Chunzheng, Zheng Yuling. Clinical application of traditional Chinese 
medicine in the treatment of postoperative complications of malignant tumors[J]. Medical Review, 2021, 27(15): 3079-3083.

[7] Jiang Pengyuan, Li Linlu, Yu Chengfeng, Mao Yun, Cai Yafang, Chu Xuelei, Zhu Shijie. Zhu Shijie's clinical experience in treating postoperative cough of lung cancer[J]. Shanghai Journal of Traditional Chinese Medicine, 2021, 55 (01): 23-26.

[8] Zheng Hongxin. Basic theory of traditional Chinese Medicine- 4th Edition[M]. Beijing: China Traditional Chinese Medicine Press, 2016.

[9] Gao Feng, Liu Jian, Guan Jumei. Song Xing's experience in treating cough with Jinkui Shenqi Pill[J]. Chinese Journal of Basic Medicine of Traditional Chinese Medicine, 2017, 23 (02): 281-282.

[10] Gao Feng, Song Xing. Song Xing's experience in treating cough with Lizhong Decoction[J]. Chinese Journal of Traditional Chinese Medicine, 2016, 31(04): 1310-1312.

[11] Wang Lihua, Zhang Yuanbing, Lan Zhizhi, Liu Liangyu. Professor Hong Guangxiang's experience in treating chronic obstructive pulmonary disease[J]. Chinese Journal of Traditional Chinese Medicine, 2016, 31 (07): 2590-2592.

[12] Chang Xing, Zhang Tian, Sui Yuyan, Zhang Qingxiang. On the clinical treatment of "spleen stomach cough" from "all diseases are born in Qi" $[\mathrm{J}]$. Chinese Journal of Basic Medicine of Traditional Chinese Medicine, 2018,24 (06): 857-859.

[13] Wang Qingnan, Lv Wenliang, Li Juanmei, Ni Yao, Hu Lingzi, Zhang Ruoxuan. Experimental research and clinical application progress of Yupingfeng Powder[J]. Chinese Journal of Traditional Chinese Medicine, 2020, 38 (09): 165-168.

[14] Wang xueru, Cheng Siyi, LV Tianyi, Li Lu, Yang Daowen. Clinical observation on the treatment of cough variant asthma with Qi deficiency, wind movement and lung stomach qi inversion syndrome by supplementing qi, dispersing wind and lowering stomach[J]. Chinese Journal of Traditional Chinese Medicine, 2020, 35 (06): 3217-3220.

[15] Liu Li, Duan Jinlin, Su Shulan, Liu Pei, Tang Yuping, Qian Dawei Research progress of Taohong Siwu Decoction, a kind of Siwu Decoction for dysmenorrhea with gynecological blood stasis syndrome[J]. Chinese Journal of Traditional Chinese Medicine, 2015, 40 (05): 814-821.

[16] Shi Yaxin, Wang Xin, Zhang Chengyuan, Zhang Xudan, Liu Shuang, Wang Yanfei, Shi Wei Network pharmacological study on Ren Mai syndrome and other diseases of Guizhi Fuling pill[J]. World Science and Technology - Modernization of Traditional Chinese Medicine, 2021, 23 (06): 1867-1877.

[17] Li Yuli, Yi Tengda, Liao Xiaonian, Yuan Zhenyi Origin and application of Shashen Ophiopogon japonicus Decoction[J]. Journal of Traditional Chinese Medicine, 2021, 49 (11): 51-57.

[18] Wang Weiguang, Li Chengwei, Han Xuejie Shen Shaogong's experience in the treatment of bronchial lung cancer[J]. Journal of Traditional Chinese Medicine, 2014, 55 (10): 822-825.

\section{Author Profile}

Lulu Liu (1995- ), female, Han nationality, from Chuzhou, Anhui Province, Graduate School of Anhui University of Traditional Chinese Medicine, professional master's degree, bachelor's degree, research direction: prevention and treatment of respiratory diseases with traditional Chinese medicine.

Corresponding author: Chen Wei (1977- ), female, Han nationality, from Tongling, Anhui Province, chief physician of respiratory department of the First Affiliated Hospital of Anhui University of Traditional Chinese Medicine, master's supervisor, doctoral degree, research direction: prevention and treatment of respiratory diseases with traditional Chinese medicine. 